\title{
VIABILIDADE TÉCNICO-ECONÔMICA DO USO DE DIFERENTES TECNOLOGIAS DE ILUMINAÇÃO PARA INDUÇÃO DE FOTOPERÍODO NA PRODUÇÃO DE MUDAS DE CRISÂNTEMO
}

\section{LUIZ A. ROSSI ${ }^{1}$, EDUARDO DAVID ${ }^{2}$, JULIANA SARUBBI ${ }^{3}$, ODAIL PAGLIARDI ${ }^{4}$}

RESUMO: Em função de suas características fisiológicas, as mudas de crisântemo necessitam de luz suplementar para evitar formação de botão floral. Isto é feito no período noturno. O presente trabalho visou a analisar a viabilidade técnico-econômica de substituir-se a atual tecnologia de iluminação artificial utilizada pelos produtores (lâmpadas incandescentes) para efeito de indução de fotoperíodo em ambiente protegido, pela tecnologia de lâmpadas de descarga, com o objetivo de reduzir o consumo de energia elétrica utilizada no processo. As lâmpadas de descarga possuem maior vida útil e apresentam menor consumo de energia quando comparadas às lâmpadas incandescentes. Os resultados das análises permitem concluir que a lâmpada fluorescente compacta integrada amarela, de $23 \mathrm{~W}$, é a que apresenta viabilidade técnica e econômica para tal substituição.

PALAVRAS-CHAVE: eficiência energética, iluminação artificial, energização rural, indução de fotoperíodo.

\section{TECHNICAL AND ECONOMICAL VIABILITY OF DISTINCT ILLUMINATION TECHNOLOGIES APPLIED TO PHOTOPERIOD INDUCTION IN CHRYSANTHEMUM PRODUCTION}

\begin{abstract}
Physiological characteristics of chrysanthemum nurseries require extra light supply to prevent buds production. That extra illumination is carried during night period. This research aimed to analyze the technical and economical viability associated to the substitution of conventional incandescent lamps used by the producers to discharge lamps to induce photoperiod, aiming electricity cost reduction in protected environment. Discharge lamps are more efficient, exhibit lower consumption and longer life when compared to the incandescent ones. The results of the analysis allow concluding that the yellow fluorescent compact integrated $23 \mathrm{~W}$ lamp turned to be technical and economically viable for the proposed substitution.
\end{abstract}

KEYWORDS: energy efficiency, artificial lighting, rural energy, photoperiod induction.

\section{INTRODUÇÃO}

O mercado mundial de flores e plantas ornamentais está em plena expansão e tem como principal exportador a Holanda, seguida pela Colômbia e Itália (MOTA et al., 2007). A floricultura empresarial brasileira vem adquirindo notável desenvolvimento nos últimos anos e caracteriza-se, já, como um dos mais promissores segmentos da horticultura intensiva no agronegócio nacional.

Observa-se, em todo o Brasil, um movimento marcado por fortes índices de crescimento da base produtiva e inclusão de novos polos geográficos regionais na produção de flores e plantas ornamentais. Segundo JUNQUEIRA \& PEETZ (2008), nos últimos anos, a atividade passou a agregar 5.152 produtores, que cultivam uma área de 8.423 hectares, cuja sustentação econômica essencial da atividade é garantida pelo vigor do mercado interno que atingiu, em 2007, a movimentação anual de US\$1,3 bilhão.

\footnotetext{
${ }^{1}$ FEAGRI/UNICAMP, Cidade Universitária Zeferino Vaz, Campinas - SP, rossi@ agr.unicamp.br

${ }^{2}$ FEAGRI/UNICAMP, Cidade Universitária Zeferino Vaz, Campinas - SP, eduardo.david@ agr.unicamp.br

${ }^{3}$ Departamento de Zootecnia, Universidade Federal de Santa Maria, CESNORS/Palmeira das Missões - RS, jusarubbi@hotmail.com

${ }^{4}$ Faculdade Municipal Professor Franco Montoro, FMPFM/Mogi Guaçu - SP, odail.pagliardi@ hotmail.com

Recebido pelo Conselho Editorial em: 28-9-2009

Aprovado pelo Conselho Editorial em: 9-8-2010
} 
O País possui, atualmente, 8 mil produtores de pequeno, médio e grande portes na produção de flores ornamentais. O principal Estado produtor é São Paulo, que responde por mais de $70 \%$ da produção nacional de flores. Em seguida, aparecem Minas Gerais, Ceará, Rio Grande do Sul e Santa Catarina. Os Estados de Alagoas, Pernambuco e Bahia já começam a ampliar a produção de flores, principalmente tropicais. No Brasil, o agronegócio da floricultura é responsável pela geração de cerca de 170 mil empregos, dos quais 84 mil (49,4\%) estão na produção, seis mil $(3,5 \%)$ relacionados à distribuição, 68 mil $(40,0 \%)$ no comércio varejista e 12 mil $(7,1 \%)$ em outras funções (PORTAL DO AGRONEGÓCIO, 2008).

O crisântemo é classificado como uma cultura sensível ao fotoperíodo e tem o florescimento induzido naturalmente em dias menores de 14,5 horas de luz (GRUSZYNSKI, 2006). Segundo ALVARENGA et al. (2003), a luz desempenha um papel importante no desenvolvimento vegetal, podendo controlar processos associados ao acúmulo de matéria seca, desenvolvimento do caule, altura, área foliar e presença de botão floral. A composição espectral da fonte de luz utilizada para indução do efeito de fotoperíodo tem influência direta no desenvolvimento dos crisântemos (REDDY et al. 1996), da mesma forma que diferentes intensidades de luz interferem no tamanho das flores (NOTHNAGL \& LARSEN, 2002).

Um dos fatores importantes ligados ao cultivo de flores é a iluminação artificial, que é responsável por cerca de $20 \%$ da energia elétrica consumida mundialmente em todas as aplicações (SEIDEL et al., 2003). Contudo, a crescente necessidade do uso de energia elétrica na agricultura e a limitação em suprir as demandas exigidas fazem com que os produtores rurais atentem para o uso racional dessa energia. Para praticar eficiência energética em um sistema produtivo, é necessário realizar auditoria energética (identificação das cargas) para a análise sistemática dos fluxos de energia em um sistema particular, visando a discriminar as perdas e a orientar programa de uso racional de insumos energéticos (MARQUES et al., 2006).

Este trabalho teve por finalidade analisar a viabilidade técnico-econômica de substituir a atual tecnologia de iluminação artificial usada pelos produtores de mudas de crisântemo, para efeito de indução de fotoperíodo em ambiente protegido, que é a tecnologia de lâmpada incandescente, pela tecnologia de descarga, com o objetivo de reduzir o consumo de energia elétrica utilizada no processo produtivo, mantendo as mesmas características produtivas, principalmente a ausência de botão floral.

\section{MATERIAL E MÉTODOS}

O experimento realizou-se em estufa de $5 \mathrm{mil} \mathrm{m}^{2}$ de empresa produtora de mudas de crisântemo, instalada no município de Mogi-Guaçu -SP, localizado nas coordenadas geográficas $22^{\circ} 34^{\prime} 23^{\prime \prime}$ de latitude e $47^{\circ} 10^{\prime} 21^{\prime \prime}$ de longitude, com clima tropical de altitude e chuvas no verão e seca no inverno, com temperatura média do mês mais quente superior a $22^{\circ} \mathrm{C}$. A empresa rural é classificada como uma unidade consumidora pertencente ao grupo A (alta tensão), subgrupo A4, apresentando uma estrutura tarifária horossazonal Verde. A empresa produz mudas de chrysanthemum sp e, para o experimento, foram cultivadas as variedades Euro Speed, Monalisa Rose, Stasteman e Papiro; todas produzidas em canteiros com 1,2 m de largura e $54 \mathrm{~m}$ de comprimento, com período de cultivo de aproximadamente 16 semanas.

No interior da estufa (vão C14), a área destinada para a realização dos ensaios tinha $54 \mathrm{~m}$ de comprimento por $6,4 \mathrm{~m}$ de largura, totalizando $345,6 \mathrm{~m}^{2}$. Dividiu-se esta área em seis parcelas iguais de $57,6 \mathrm{~m}^{2}$, instalando-se em cada parcela apenas um tipo de lâmpada. As lâmpadas utilizadas no experimento e denominadas de tratamentos $(\mathrm{T})$ foram: T1: lâmpada incandescente de $100 \mathrm{~W}$; T2: lâmpada fluorescente compacta integrada branca de ${ }^{23} \mathrm{w}$; T3: lâmpada fluorescente tubular de $40 \mathrm{~W}$; T4: lâmpada de vapor de mercúrio de $125 \mathrm{~W}$; T5: lâmpada de vapor de sódio de alta pressão de $70 \mathrm{~W}$, e T6: lâmpada fluorescente compacta integrada amarela de $23 \mathrm{~W}$. Na Figura 1 , ilustra-se a disposição dos tratamentos no experimento. 
As lâmpadas de descarga utilizadas no experimento possuem as seguintes temperaturas de cor, em graus Kelvin: lâmpada incandescente de $100 \mathrm{~W}: 2.700 \mathrm{~K}$; lâmpada fluorescente compacta integrada branca de $23 \mathrm{~W}$ : $6.500 \mathrm{~K}$; lâmpada fluorescente tubular de $40 \mathrm{~W}$ : $5.000 \mathrm{~K}$; lâmpada de vapor de mercúrio de $125 \mathrm{~W}: 4.100 \mathrm{~K}$; lâmpada de vapor de sódio de $70 \mathrm{~W}$ de alta pressão: $1.950 \mathrm{~K}$; lâmpada fluorescente compacta integrada amarela de $23 \mathrm{~W}: 2.700 \mathrm{~K}$.

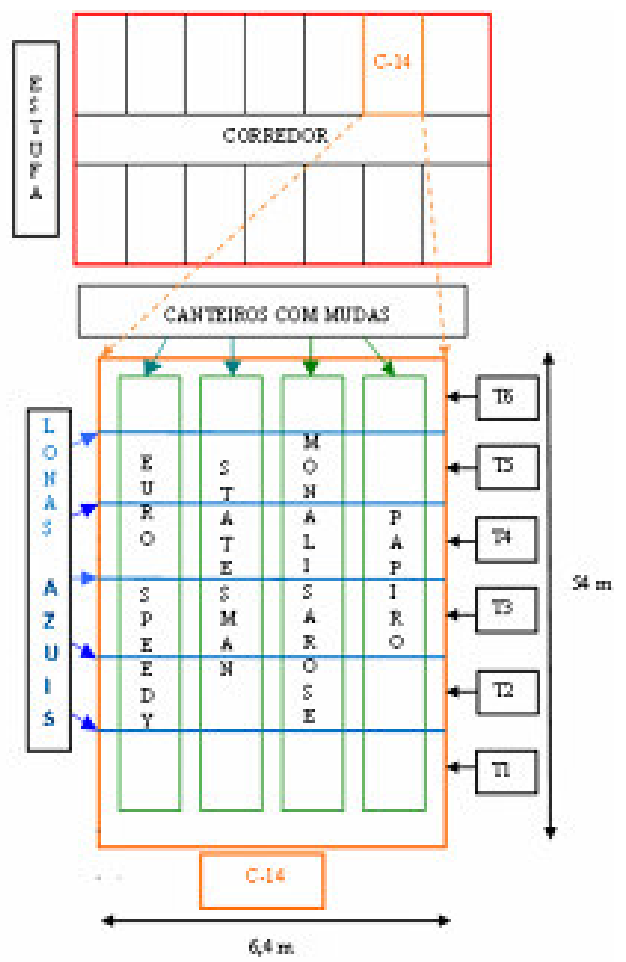

FIGURA 1. Disposição dos tratamentos no experimento. Distribution of the treatments in the experiment.

\section{Parâmetros do uso de energia elétrica}

O método utilizado para o cálculo do número de luminárias por tratamento foi o Método dos Lumens Médios ou Método da Iluminância Média Geral, função da necessidade da uniformização da intensidade luminosa ou iluminância sobre a cultura (CREDER, 2002). Na Tabela 1, mostra-se a vida útil das lâmpadas utilizadas e a quantidade calculada no processo produtivo.

TABELA 1. Vida útil e número de lâmpadas utilizado no processo produtivo. Shelf Life and number of lamps used in the production process.

\begin{tabular}{crrr}
\hline \multirow{2}{*}{ Tratamento } & \multicolumn{2}{c}{ Vida Útil } & \multicolumn{2}{c}{$\begin{array}{c}\text { Número de } \\
\text { Lâmpadas }\end{array}$} \\
\cline { 2 - 3 } & Em horas & Em anos & 12 \\
T1 & 1.000 & 1,72 & 24 \\
T2 & 10.000 & 13,73 & 12 \\
T3 & 8.000 & 12,84 & 6 \\
T4 & 16.000 & 17,16 & 6 \\
T5 & 24.000 & 48,04 & 12 \\
T6 & 10.000 & 13,73 & \\
\hline
\end{tabular}

Fonte: Serviço Philips de Orientação Técnica. Site: www.luz.philips.com (visitado em 5-3-2009).

Por meio de pesquisa em empresas comerciais de materiais elétricos, obtiveram-se os custos de instalação (investimento inicial) para todos os tipos de lâmpadas usadas no experimento, cujos detalhes estão na Tabela 2. 
TABELA 2. Custo total de instalação de cada sistema de iluminação (tratamento). Total installation cost for each lighting system (treatment).

\begin{tabular}{cccccc}
\hline \multirow{2}{*}{ Tratamento } & \multicolumn{2}{c}{ Lâmpadas } & \multicolumn{2}{c}{ Reatores } & Investimento \\
\cline { 2 - 5 } & Quantidade & Custo (R\$) & Quantidade & Custo (R\$) & Inicial (R\$) \\
\hline T1 & 12 & 3,66 & ---- & ---- & 43,92 \\
T2 & 24 & 12,03 & ---- & ---- & 288,72 \\
T3 ${ }^{1}$ & 12 & 11,60 & 12 & 30,44 & 504,48 \\
T4 & 6 & 9,71 & 6 & 36,28 & 275,94 \\
T5 & 6 & 13,29 & 6 & 33,93 & 283,32 \\
T6 & 12 & 12,03 & ---- & ---- & 144,36 \\
\hline
\end{tabular}

Fonte: Empresa Nortel Ltda. (9-5-2009).

Os parâmetros de energia elétrica coletados por medidores multivariáveis de energia elétrica, modelo SAGA 2300 - 1387 da marca ESB, foram a demanda $(\mathrm{kW})$, o consumo $(\mathrm{kWh})$ e o fator de potência (adimensional). Por meio dos dados coletados da energia elétrica, realizou-se a comparação das médias horárias de demanda de potência $(\mathrm{kW})$, de consumo de energia elétrica $(\mathrm{kWh})$, de fator de potência e de consumo específico entre as lâmpadas testadas, por meio de Análise de Variância e Teste Tukey, a 5\%. O regime de acionamento do sistema de iluminação artificial inicia-se às $22 \mathrm{~h} 30 \mathrm{~min}$ de um dia e termina às $4 \mathrm{~h} 10 \mathrm{~min}$ do dia seguinte, com intervalos de 10 minutos ligados por 20 minutos desligados, totalizando duas horas de acionamento diário.

\section{Parâmetro do vegetal: presença de botão floral}

Segundo VERMEULEN \& SCHOUTSEN (2006), um dos indicadores produtivos em mudas de crisântemo é a ausência de botão floral. Este parâmetro de produção mede a presença de botão floral nas mudas produzidas, o que não é desejável. A intenção do uso de iluminação artificial no período noturno, para efeito de indução de fotoperíodo, é a da ausência total de botão floral, o que não é possível para todas as variedades de crisântemo, inclusive as cultivadas neste experimento, como, por exemplo, o Papiro, que é um Garden mum utilizado para fazer arranjos florais. O produtor utiliza o método usual de porcentagem, ou seja, o número de mudas que apresentaram botão floral em relação ao número de mudas plantadas por meio de amostragem.

\section{Métodos de análise de viabilidade econômica}

A análise de viabilidade econômica é necessária para apoiar, na tomada de decisões, os gestores na realização ou não de um determinado investimento. Para identificar qual o sistema de iluminação mais viável do ponto de vista econômico para substituir o sistema de lâmpadas incandescentes, usaram-se os conceitos tradicionais de análise, que são o valor presente líquido, a taxa interna de retorno (TIR) e o período de retorno do capital (payback) (KOPTTKE \& CASAROTTO FILHO, 2000; MARQUES et al., 2006; HIRSCHFELD, 1992; BALARINE, 2004; MARINI \& ROSSI, 2005).

O valor presente líquido (VPL) é bastante interessante quando se deseja comparar alternativas mutuamente excludentes. Todos os custos e benefícios ocorridos ao longo do período estudado são trazidos ao valor presente. A alternativa que oferecer o maior valor presente líquido será considerada, por esse critério, a mais atraente. Em se tratando de analisar alternativas com vidas úteis diferentes, lança-se mão do conceito de reposição contínua, ou seja, as tecnologias serão repostas indefinidamente. Alguns autores denominam essa análise de valor anual líquido.

Outro critério muito usado é a taxa interna de retorno (TIR) de um projeto. Trata-se da taxa de desconto do fluxo de caixa que anula o valor líquido presente, ou anual, do empreendimento, dentro

\footnotetext{
${ }^{1}$ Obs. Cada lâmpada fluorescente tubular custa $\mathrm{R} \$ 3,22$ e exige luminária que custa $\mathrm{R} \$ 5,98$ por unidade e $\mathrm{R} \$ 2,40$ dos soquetes de fixação da lâmpada na luminária. Assim, o conjunto luminária, lâmpada e soquete tem valor unitário de R \$11,60.
} 
de um período de tempo estipulado. Calculada a taxa de desconto, TIR, do projeto, deve-se compará-la a uma taxa de juros de referência, que pode ser a taxa de atratividade do setor (no caso do mercado de crisântemos, de $15 \%$ anuais) ou mesmo ser uma taxa de juros do mercado financeiro. Se a TIR, em questão, for maior que a taxa de atratividade, o projeto é atraente, segundo esse critério de análise.

A desvantagem desse método, TIR, em relação à análise do valor presente líquido, é que, embora ofereça a taxa de retorno do empreendimento, nada garante que o retorno financeiro obtido em cada período seja reinvestido à mesma taxa da TIR.

O terceiro critério de decisão é o tempo de retorno do capital (payback). Trata-se de um critério muito utilizado para estimar o tempo de retorno do capital investido, devido sua simplicidade de aplicação. Basta dividir o custo da implantação do empreendimento pelo benefício auferido para se ter o payback simples (não descontado). Neste caso, não se está considerando o custo de capital, ou seja, a taxa de atratividade do projeto. Ao considerar o payback descontado, observa-se o real valor do dinheiro no tempo, obtendo uma estimativa financeira mais realista.

Os fluxos de caixa de substituição da tecnologia usada (T1), pelas tecnologias de lâmpadas de descarga foram confeccionadas considerando o horizonte de 48 anos devido ser a vida útil da lâmpada de sódio (T5) a maior entre as tecnologias utilizadas, conforme Tabela 1.

Neste horizonte temporal, como as lâmpadas incandescentes devem ser trocadas a cada 1,72 ano, escolhem-se períodos desse comprimento de tempo, totalizando 28 períodos. Portanto, as lâmpadas de sódio (T5) são trocadas uma única vez ( $28^{\circ}$ período), assim como as de mercúrio (no $16^{\circ}$ período), porém as de flúor (T3) trocam-se três vezes $\left(8^{\circ}, 16^{\circ}\right.$ e $24^{\circ}$ períodos) e as compactas (T2 e T6), duas vezes ( $10^{\circ}$ e $20^{\circ}$ períodos). Não estão previstas reposições para os reatores dentro do prazo considerado.

Os custos de manutenção são desconsiderados, pois estes podem ser efetuados pelos próprios funcionários devido à simplicidade de operação (praticamente, simples troca de lâmpadas). $\mathrm{O}$ valor presente líquido foi calculado considerando uma taxa de atratividade de $15 \%$ anuais, equivalentes a 27,17\% no período de 1,72 ano, representativa do mercado de crisântemo.

\section{RESULTADOS E DISCUSSÃO}

A ausência de botões florais é o aspecto produtivo mais importante, ligado diretamente ao controle fotoperiódico, sendo parâmetro decisivo na avaliação das tecnologias testadas. Para as variedades analisadas, os resultados da Tabela 3 mostram que todos os tratamentos tiveram o mesmo comportamento que o tratamento-testemunha T1.

Embora exista diferença estatística entre os tratamentos, no uso de iluminação artificial para indução de fotoperíodo em mudas de flores, o principal resultado é se há ou não presença de botão floral. As variedades Papiro e Statesman são utilizadas em arranjos florais por apresentarem botões florais já na fase de muda.

TABELA 3. Resultado da análise do parâmetro vegetal presença de botão floral, em \%. Analysis results of bud presence as plant parameter (percentage).

\begin{tabular}{lcccccc}
\hline \multicolumn{7}{c}{ Tratamento } \\
\hline Variedade & $\mathrm{T} 1$ & $\mathrm{~T} 2$ & $\mathrm{~T} 3$ & $\mathrm{~T} 4$ & $\mathrm{~T} 5$ & $\mathrm{~T} 6$ \\
\hline Papiro & $49,5 \mathrm{ab}$ & $43,3 \mathrm{a}$ & $51,8 \mathrm{~b}$ & $55,6 \mathrm{~b}$ & $42,6 \mathrm{a}$ & $55,2 \mathrm{~b}$ \\
Euro Speedy & 0 & 0 & 0 & 0 & 0 & 0 \\
Mona Lisa & 0 & 0 & 0 & 0 & 0 & 0 \\
Statesmann & $3,3 \mathrm{a}$ & $6,9 \mathrm{c}$ & $5,9 \mathrm{bc}$ & $4,8 \mathrm{ab}$ & $4 \mathrm{a}$ & $4,7 \mathrm{ab}$ \\
\hline a,b,c
\end{tabular}

$\overline{\mathrm{a}, \mathrm{b}, \mathrm{c}}$. Médias seguidas de letras diferentes na mesma linha diferem ( $\mathrm{p}>0,05)$, pelo teste de Tukey. 
Como descrito em material e métodos, os parâmetros de energia coletados foram a demanda, o consumo e o fator de potência, cujos resultados foram analisados por meio de Análise de Variância e teste Tukey, a 5\%, e agora apresentados na Tabela 4.

TABELA 4. Resultados das análises dos parâmetros de energia. Analysis results of energy parameters.

\begin{tabular}{lcccccc}
\hline \multicolumn{7}{c}{ Tratamento } \\
\hline Parâmetro & T1 & T2 & T3 & T4 & T5 & T6 \\
\hline Demanda $(\mathrm{kW})$ & $1,258 \mathrm{c}$ & $0,768 \mathrm{~b}$ & $0,484 \mathrm{ab}$ & $0,767 \mathrm{~b}$ & $0,528 \mathrm{ab}$ & $0,327 \mathrm{a}$ \\
Consumo $(\mathrm{kWh})$ & $15.138,00 \mathrm{~b}$ & $7.243,00 \mathrm{a}$ & $5.526,00 \mathrm{a}$ & $8.593,00 \mathrm{a}$ & $5.432,00 \mathrm{a}$ & $3.701,00 \mathrm{a}$ \\
Fator de potência & $1,0 \mathrm{~b}$ & $0,56 \mathrm{a}$ & $0,98 \mathrm{~b}$ & $0,95 \mathrm{~b}$ & $0,95 \mathrm{~b}$ & $0,56 \mathrm{a}$ \\
\hline a,b,c, Médias seguidas de letras diferentes na mesma linha diferem $(\mathrm{p}>0,05)$, pelo teste de Tukey.
\end{tabular}

Pela Tabela 4, tanto em relação à demanda quanto ao consumo, a análise estatística mostra que a tecnologia de alta intensidade de descarga (HID), tratamentos T2 a T6, difere daquela de filamento incandescente, tratamento T1. Embora esta análise demonstre não haver diferenças significativas entre os tratamentos T3, T5 e T6 (menores valores de demanda) e entre T2, T3, T4, T5 e T6 (menores valores de consumo), os dados da Tabela 2 apontam que o melhor resultado é o do tratamento T6, por apresentar os menores valores de demanda e de consumo. Isto coincide quando se faz a análise sobre o uso eficiente da energia elétrica, para o qual o resultado mais significativo são os menores valores de demanda de potência e de consumo, e o maior valor de fator de potência. O tratamento T6 atendeu aos dois primeiros requisitos, e o tratamento T1 (testemunha) atendeu ao terceiro, tal qual observado por JACOME \& ROSSI (2009), comparando diferentes sistemas de iluminação artificial na produção de ovos de postura.

Na Tabela 5, apresentam-se os dados de consumo mensal das tecnologias utilizadas. Ainda, são apresentados os custos mensais de energia elétrica dos diferentes sistemas de iluminação. Tendo em vista que o grupo tarifário é A (alta tensão), classe Empresa Rural, com enquadramento na Tarifa Verde, no qual a tarifa de consumo de energia elétrica é, em média, de R $\$ 0,19353 / \mathrm{kWh}$ (CPFL - preço de 2009), o custo mensal do sistema é calculado pela eq.(1):

Custo mensal por sistema $=$ consumo mensal por sistema $\mathrm{x}$ tarifa por consumo

TABELA 5. Consumo (kWh mês ${ }^{-1}$ ) e custo mensal e anual (R\$) de energia elétrica por tratamento. Consumption (kWh/month), monthly and annual electricity cost (R\$) per treatment.

\begin{tabular}{|c|c|c|c|}
\hline \multirow{2}{*}{ Tratamento } & \multirow{2}{*}{ Consumo (kWh mês $\left.{ }^{-1}\right)$} & \multicolumn{2}{|c|}{ Custo } \\
\hline & & Mensal - R\$ mês ${ }^{-1}$ & Anual - R\$ ano ${ }^{-1}$ \\
\hline $\mathrm{T} 1$ & 72,00 & 13,93 & 167,21 \\
\hline $\mathrm{T} 2$ & 40,32 & 7,80 & 93,64 \\
\hline $\mathrm{T} 3$ & 32,40 & 6,27 & 75,24 \\
\hline $\mathrm{T} 4$ & 50,04 & 9,68 & 116,21 \\
\hline T5 & 30,24 & 5,85 & 70,23 \\
\hline T6 & 20,16 & 3,90 & 46,82 \\
\hline
\end{tabular}

Fonte: Edição própria

De acordo com as Tabelas 2 e 5, comparando-se apenas as lâmpadas de descarga, o sistema de iluminação que apresenta maior investimento inicial é o da lâmpada fluorescente tubular de $40 \mathrm{~W}$ (T3) e o de menor investimento é a fluorescente compacta integrada amarela, de $23 \mathrm{~W}$ (T6). 


\section{Análise de viabilidade econômica}

Os resultados da análise econômica dos fluxos de caixa de todos os tratamentos são apresentados na Tabela 6, ou seja, os valores do VPL, da TIR e do Payback dos sistemas de iluminação artificial com lâmpadas de descarga, quando comparados com o sistema com lâmpadas incandescentes (testemunha, T1).

TABELA 6. Resultados dos métodos de avaliação da substituição da lâmpada incandescente (T1) pelas de tecnologia de alta intensidade de descarga ${ }^{2}$. Evaluation methods results of replacing incandescent lamp (T1) by the high intensity discharge technology lamps.

\begin{tabular}{|c|c|c|c|c|}
\hline \multirow[b]{2}{*}{ Substituição de Tecnologias } & \multirow[b]{2}{*}{ TIR } & \multirow{2}{*}{$\begin{array}{c}\text { Valor } \\
\text { Presente } \\
\text { Líquido (R\$) }\end{array}$} & \multicolumn{2}{|c|}{ Payback Descontado } \\
\hline & & & Período & Ano \\
\hline Incandescente $\mathrm{x}$ compacta branca (T2) & $69,21 \%$ & 780,00 & 1,76 & 3,03 \\
\hline Incandescente x flúor (T3) & $55,97 \%$ & 942,00 & 2,25 & 3,87 \\
\hline Incandescente x mercúrio (T4) & $54,08 \%$ & 609,00 & 2,35 & 4,04 \\
\hline Incandescente x sódio (T5) & $88,03 \%$ & $1.137,00$ & 1,35 & 2,32 \\
\hline Incandescente x compacta amarela(T6) & $249,87 \%$ & $1.484,00$ & 0,46 & 0,79 \\
\hline
\end{tabular}

A substituição das lâmpadas incandescentes (T1) pelas diversas lâmpadas de descarga apresenta elevadas taxas internas de retorno para o período considerado, sendo que a fluorescente compacta integrada amarela, de $23 \mathrm{~W}$ (T6), é a de maior valor $(249,87 \%)$. Ainda, o valor presente líquido desta do tratamento T6 é o maior entre as alternativas propostas (R\$1.484,00), e seu payback descontado é, também, o mais atraente, mostrando que o tempo de retorno do investimento inicial é inferior a um ano.

\section{CONCLUSÕES}

As variedades Euro Speedy e Mona Lisa mostraram-se indiferentes ao tipo de tecnologia utilizada na iluminação, não apresentando botão floral. Por outro lado, a presença de botão floral nas variedades Statesman e Papiro, observada com o uso da lâmpada fluorescente compacta integrada amarela, de $23 \mathrm{~W}$, não diferiu significativamente da lâmpada incandescente de $100 \mathrm{~W}$. Na variedade Papiro, a lâmpada fluorescente compacta integrada branca, de $23 \mathrm{~W}$, e a lâmpada de vapor de sódio, de $70 \mathrm{~W}$, de alta pressão foram as que apresentaram as menores porcentagens de botão floral. Contudo, na variedade Statesman, ocorre diferença significativa entre a lâmpada fluorescente compacta integrada amarela, de $23 \mathrm{~W}$, e a fluorescente compacta integrada branca, de $23 \mathrm{~W}$, favorecendo a lâmpada fluorescente compacta amarela, de $23 \mathrm{~W}$, que, por sua vez, não difere da lâmpada incandescente de $100 \mathrm{~W}$, que possui a menor presença de botão floral.

Associado ao resultado técnico, ao se analisarem os critérios tradicionais de análise de viabilidade econômica, houve total favorecimento ao sistema com lâmpadas fluorescentes compactas integradas amarelas, com retorno do investimento em aproximadamente nove meses e quinze dias, justificando plenamente sua implantação.

\section{AGRADECIMENTOS}

Ao FAEPEX/UNICAMP, pelo apoio financeiro ao trabalho, e ao CNPq, pelo apoio na forma de bolsa de mestrado.

\footnotetext{
${ }^{2}$ Obs.: Os valores referem-se a períodos de 1,72 ano e, portanto, o valor presente líquido, cuja taxa de desconto é de $15 \%$ a.a., tem taxa equivalente $27,17 \%$ por período.
} 


\section{REFERÊNCIAS}

ALVARENGA, A.A.; CASTRO, E.M.; LIMA JÚNIOR, E.C.; MAGALHÃES, M.M. Effects of different light levels on the initial growth and photosynthesis of Croton urucurana Baill. in southeastern Brazil. Revista Árvore, Viçosa - MG, v.27, n.1, p.53-57, 2003.

BALARINE, O.F.O. Tópicos de matemática financeira e engenharia econômica. Porto Alegre: EDIPUCRS, 2004. 77 p.

CREDER, H. Instalações elétricas. 14.ed. Rio de Janeiro: LTC, 2002. 479 p.

GRUSZYNSKI, C. Produção de crisântemos. Disponível em: <http://www.emater.tche.br/ docs/ agricultura/crisa/crisant.htm>. Acesso em: 12 jan. 2006.

HIRSCHFELD, H.E. Engenharia econômica e análise de custos. São Paulo: Atlas, 1992. 461 p.

JACOME, I.M.D.T.; ROSSI, L.A. Diferentes sistemas de iluminação artificial usados no alojamento de poedeiras leves. 2009. 120 f. Tese (Doutorado) - Faculdade de Engenharia Agrícola, Universidade Estadual de Campinas, Campinas, 2009.

JUNQUEIRA, A.H.; PEETZ, M.S. Mercado interno para os produtos da floricultura brasileira: características, tendências e importância socioeconômica recente. Revista Brasileira de Horticultura Ornamental, Campinas, v.14, n.1, p.37-52, 2008.

KOPITTKE, H.B.; CASAROTTO FILHO, N. Análise de investimentos. São Paulo: Atlas, 2000. 467 p.

MARINI, J.A.; ROSSI, L.A. Sistematização do dimensionamento técnico e econômico de sistemas fotovoltaicos isolados por meio de programa computacional. Engenharia Agrícola, Jaboticabal, v.25, n.1, p.67-75, 2005.

MARQUES, M.; HADDAD, J.; MARTINS, A.R.S. Conservação de energia: eficiência energética e instalações. Itajubá: Fundação de Pesquisa e Assessoramento à Indústria, Universidade Federal de Itajubá, 2006. 597 p.

MOTA, P.R.D.; BOAS, R.L.V.; SOUSA, V.F.; RIBEIRO, V.Q. Desenvolvimento de plantas de crisântemo cultivadas em vaso em resposta a níveis de condutividade elétrica. Engenharia Agrícola, Jaboticabal, v.27, n.1, p.164-171, 2007.

NOTHNAGL, M.; LARSEN, R.U. The relationship between chrysanthemum flower diameter and light condition in the greenhouse - A modeling approach. Acta Horticulture, Wageningen, v.593, p.179-184, 2002.

PORTAL DO AGRONEGÓCIO. Produção de flores e plantas ornamentais aumenta no Brasil.

Disponível em: <http://www.portaldoagronegocio.com.br/conteudo.php?id=24701>. Acesso em: 15 set. 2008.

REDDY, V.K.; RAJAPAKSE, N.C.; YOUNG, R.E. The influence of spectral composition growth and development of Chrysanthemum plants. Acta Horticulture, Wageningen, v.440, p.292-297, 1996.

SEIDEL, Á.R.; BISOGNO, F.E.; PINHEIRO, H. Reator eletrônico auto-oscilante com controle de intensidade luminosa. SBA - Controle \& Automação, Porto Alegre, v.14, n.2, p.78-81, 2003.

VERMEULEN, M.; SCHOUTSEN, R. Kwaliteitshandboek stekproductie Dekker Chrysanten B.V. $3^{\text {rd }}$ ed: Hensbroek: Dekker De Witt, 2006. p.25-32 (Boletim) 